\title{
A new perspective to gynecological cancer treatment
}

\section{Opinion}

According to the World Health Organization, cancer is defined as process of growth of abnormal cells beyond their boundaries that can invade other parts of the body or spread to other organs. ${ }^{1}$ Ever since the development of cancer therapies, a major problem has been the array of side effects and complications of chemotherapy, which may include hair loss, diarrhea, as well as life threatening fever and neutropenia and even tumor lysis syndrome. As of now, chemotherapy has been a synonym of side effects and patient suffering. What if we could find a way to selectively kill cancer cells while leaving healthy cells unharmed? What if we could change the actual paradigm where chemotherapy means side effects? The wait might not be long to reach that goal. Herbolary and phytotherapy have accompanied medicine for centuries, and many of the drugs used in today's medicine are derived from plants. Such is the case of artemisinin, a compound derived from the Artemisia annua plant. This plant has been widely used by the Chinese since the Jin dynasty around 317-420 AD due

to its medicinal properties specifically for reducing fever. ${ }^{2}$ It was later identified as a viable therapy against the Plasmodium parasite, and is now considered one of the first-choice therapies for malaria. ${ }^{2}$ After several years of studying the compound and its synthetic derivatives, researchers found that it possesses strong antineoplastic capabilities with surprisingly minimal toxicity and side effects. ${ }^{3}$ As of this date, studies with this compound have been conducted both in vivo and in vitro with promising results.

Specifically in what concerns to the gynecologic malignancies, there are reports of the drug's effectiveness against in vitro breast and ovary cancer cells. ${ }^{4}$ In a study conducted with cervical carcinoma patients who were treated with dihydroartemisinin, they observed an improvement in the signs and symptoms of the disease as well as remission in 8 of 10 patients. ${ }^{5}$ In a review by Goodrich and colleagues, it is also asserted that artemisinin has been effective against HPV-infected cervical cells with cancerous transformation. ${ }^{6}$ The previously citations are just a small sample of a vast number of the reported clinical experience with this compound. Although there are currently no approved regimes with this drug, it seems as it is just a matter of time before the use of artemisinin and its derivatives is expanded and worldwide recognized. The effectivity of the drug along with its minimal side effects would change the current paradigm of chemotherapy.
Volume 7 Issue 2 - 2018

\section{Enrique Konstat-Korzenny, Rosalino Vázquez-López}

Departamento de Microbiología del Centro de Investigación en Ciencias de la Salud (CICSA), Facultad de Ciencias de la Salud, Mexico

Correspondence: Enrique Konstat-Korzenny, Departamento de Microbiología del Centro de Investigación en Ciencias de la Salud (CICSA) Facultad de Ciencias de la Salud, Universidad Anáhuac México Campus Norte. Av. Universidad Anáhuac 46 Col. Lomas Anáhuac Huixquilucan, Estado de México 52786, México,Tel 52 55205264, Email enriquekonstat@gmail.com

Received: March 07, 2018| Published: March 16, 2018

\section{Acknowledgement}

None.

\section{Conflict of interest}

None.

\section{References}

1. http://www.who.int/cancer/en/

2. Tu Y. Artemisinin-A Gift from Traditional Chinese Medicine to the World (Nobel Lecture). Angew Chem Int. 2016;55(35),10210-10226.

3. Konstat-Korzenny E, Ascencio-Aragón JA, Niezen-Lugo S, et al. Artemisinin and Its Synthetic Derivatives as a Possible Therapy for Cancer. Med Sci (Basel). 2018;6(1),19.

4. Efferth T, Dunstan H, Sauerbrey A, et al. The anti-malarial artesunate is also active against cancer. Int J Oncol. 2001;18(4),767-773.

5. Jansen FH, Adoubi I, JC KC, et al. First study of oral Artenimol-R in advanced cervical cancer: Clinical benefit, tolerability and tumor markers. Anticancer Res. 2011;31(12),4417-4422.

6. Goodrich SK, Schlegel CR, Wang G, et al. Use of artemisinin and its derivatives to treat HPV-infected/transformed cells and cervical cancer: a review. Future Oncol. 2014;10(4),647-654. 